Intersections

Canadian Journal of Music

Revue canadienne de musique
Intersections

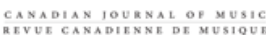

\title{
Fonctionnalisme technologique en musique
}

\section{Dragos Chiriac}

Volume 35, numéro 1, 2015

URI : https://id.erudit.org/iderudit/1038942ar

DOI : https://doi.org/10.7202/1038942ar

Aller au sommaire du numéro

\section{Éditeur(s)}

Canadian University Music Society / Société de musique des universités canadiennes

ISSN

1911-0146 (imprimé)

1918-512X (numérique)

Découvrir la revue

Citer cet article

Chiriac, D. (2015). Fonctionnalisme technologique en musique. Intersections, 35(1), 3-26. https://doi.org/10.7202/1038942ar

\section{Résumé de l'article}

Dans le jargon philosophique habituel, un jugement de type « universel » est un concept toujours valide dans toutes les situations / époques, qui s’applique à tous les individus qu'il a la prétention de catégoriser. Au cours de cet article, nous suggérons que l'ébauche d'une quelconque définition " universelle " de l'art doit se heurter à des problèmes ontologiques (l'essence de l'art) sérieux. Nous proposons qu'il soit plus prudent de s'abstenir de faire de tels jugements en ayant une approche fonctionnaliste pour décrire sans définir le processus créatif ainsi que les oeuvres qui y aboutissent. Nous faisons la démonstration de cette approche construite sur un refus conscient de définir l'art, en analysant (avec les catégories des fins et des moyens) le rapport à la technologie du pianiste Glenn Gould. Il s'agit, en quelque sorte, d'un dialogue ouvert avec le lecteur. Finalement, nous aboutissons à la conclusion qu'il peut être pertinent pour le chercheur-créateur (un mélange entre le théoricien et l'artiste qui cherche à valider son art) d'opter pour une esthétique singulière ou d'essayer de constituer une théorie universelle de l'art en attaquant le problème ontologique par le biais de la linguistique (une suggestion, sans garanties). En dehors de notre approche fonctionnaliste, qui par rigueur intellectuelle peut tendre vers un relativisme insatisfaisant, il s'agit des seuls choix prometteurs qui ne se limitent pas aux analyses historiques ou aux analyses d'oeuvres individuelles (le paradigme actuel de l'enseignement théorique universitaire en musique) auxquels nous avons pensé.
Copyright @ Canadian University Music Society / Société de musique des universités canadiennes, 2016
Ce document est protégé par la loi sur le droit d'auteur. L’utilisation des services d'Érudit (y compris la reproduction) est assujettie à sa politique d'utilisation que vous pouvez consulter en ligne.

https://apropos.erudit.org/fr/usagers/politique-dutilisation/ 


\title{
FONCTIONNALISME TECHNOLOGIQUE EN MUSIQUE
}

\author{
Dragos Chiriac
}

\section{INTRODUCTION}

Le présent article s'adresse autant aux créateurs qu'aux chercheurs, philosophes et musicologues, intéressés par la musique et l'enregistrement, et son but est double. D'une part, on y discute de l'enregistrement comme finalité et comme moyen (il s'agit d'une approche fonctionnaliste) dans le processus de création d'un objet musical. D'autre part, et de manière plus générale, il s'agit également de l'occasion pour nous de présenter une propédeutique pour un cadre logique de discussion non dogmatique en esthétique. À notre avis, ces deux propos aux ramifications logiques communes, doivent nécessairement être abordés en même temps, car il existe de nombreuses définitions contradictoires et des principes théoriques arbitraires préétablis (plusieurs d'ordre éthique) qu'il nous faut exposer et remettre en question pour examiner sans préjugés le rôle théorique et pratique que peut avoir l'enregistrement audio pour le musicien et sa musique. Par souci de clarté pour l'application de notre cadre logique de discussion, nous prenons pour exemple Glenn Gould (1932-1982) : à notre avis, le pianiste a un rapport pragmatique à la technologie, qui est sympathique au nôtre, dans la réalisation phonographique ${ }^{1}$ de sa musique.

Gould adorait le contrôle et n'affectionnait pas vraiment la salle de concert; il n'aimait pas l'idée de se commettre, de la première note à la dernière note d'une pièce, à la performance du moment et à ses contingences que les conditions humaines et naturelles nécessitent ${ }^{2}$ : «It [technology] has the capability of replacing those awful and degrading and humanly damaging uncertainties which the concert brings with it; it takes the specific personal performance information out of the musical experience ${ }^{3}$ ", affirme-t-il en 1981. Le pianistecompositeur avait le trac en concert et se retira complètement de la scène en $1964^{4}$. Nous avons choisi d'écarter une venue psychologisante pour interpréter ce choix, favorisant plutôt une approche synthétisable des idées du pianiste, afin de pouvoir réfléchir à leur valeur intellectuelle en dehors de leur portée locale et biographique.

1 L'ensemble des étapes de réalisation d'un ou de plusieurs enregistrements constituant un album.

Ostwald 1997, p. 91.

Gould 1990, p. 452.

4 Mantere 2006, p. 132-414. 
En somme, et si nous n'enlevons pas toute forme d'intentionnalité à Gould, nous croyons que ce dernier avait une vision puriste de la musique et de ses modes d'actualisation, en tant qu'ils devaient tendre vers son idéal de perfection, grâce aux potentiels technologiques permettant de synthétiser une infinité d'interprétations. Nous sommes ici en présence d'une idée esthétique et d'un "positivisme technologique» qui méritent qu'on les examine. Nous faisons usage de catégories logiques utilitaristes ${ }^{5}$, car elles offrent un cadre logique simple, rigoureux et applicable in concreto en faisant la distinction entre les fins, les moyens, les causes, les effets et les corrélations. Les catégories utilitaristes (celles des fins et des moyens en particulier), permettent également de mieux comprendre le fonctionnement de la création d'un objet musical ${ }^{6}$, sans devoir se placer / commettre ontologiquement sur la phonographie, la performance, la fidélité et l'authenticité, qui sont des catégories situées au cœur des incessantes divisions dogmatiques entre les arts considérés comme majeurs et ceux que l'on met parfois de côté dans les hauts cercles académiques: les arts mineurs.

Cette préférence d'une description fonctionnaliste sur l'ontologie nous vient d'un principe épistémologique subjectif selon lequel l'essence des choses n'est pas sujette à être théorisée ou formalisée sans grands risques de commettre des erreurs en raison de sa nature non évidente, alors que le fonctionnement des choses (ou leur relation entre elles pour nous-mêmes), lui, est observé et construit par l'homme, dans un monde vécu de manière subjective, sans devoir nécessairement se positionner idéologiquement sur la nature des choses dont on observe la relation. Des axiomes similaires sont également présents dans certaines phénoménologies de la perception, où les phénomènes sont le produit du déploiement de notre conscience. Un examen phénoménologique devient alors nécessairement un examen de conscience où la question de l'existence même du monde peut être mise en suspens, laissant néanmoins un malaise ontologique proportionnel à la difficulté du sujet étudié quand il est question de la conscience, du corps et du cerveau, et ce, même lorsqu'on les considère, en dehors de leur matérialité, comme faisant partie de l'interface générale qui permet notre expérience du monde. Avec un fonctionnalisme, plus sobre et peut-être pour cette raison moins fascinant, nous avons l'avantage de concéder que la relation entre les choses est le fruit d'une rationalisation. Autrement dit, les relations entre les choses ne sont que des mots (il s'agit d'un principe nominaliste) que nous utilisons pour mieux fonctionner ou pour nous comprendre entre nous dans nos vies mortelles d'hommes. Nous acceptons donc que les relations entre les choses soient elles-mêmes très relatives et qu'elles dépendent du temps, de la société, de notre perception, des lois législatives des gouvernements, etc.

Par contre, notre fonctionnalisme ne se tire pas d'affaire aussi facilement, car les gênes de l'objectivité, de la perception et de l'ontologie se régressent

5 Nous évitons personnellement d'associer l'art à l'éthique, bien que ces associations soient courantes dans de nombreux discours esthétiques que nous ne partageons pas.

6 Nous utilisons ce terme de manière très générale sans en donner une définition précise (fonctionnelle ou identitaire), afin que nous n'amputions pas de catégories musicales que nous pouvons avoir oubliées. 
jusqu’aux mots. Plusieurs mots demeurent très problématiques à définir, et cela est spécialement le cas pour l'art ainsi que ses sous-domaines, qui évoluent constamment, annexent des disciplines, acquièrent des nouvelles fonctions, puis nient des anciennes, etc. Les nombreux essais auxquels nous avons été exposés en esthétique sont toujours sujets à des réfutations de leurs fondements instables et parviennent ordinairement à des échecs lamentables, bien que leurs efforts puissent être louables: certaines définitions trop restrictives sont empreintes d'une vision priorisée (une division hiérarchique corps-esprit, par exemple) de l'être humain qui ne rend pas nécessairement justice à certains arts sous-valorisés, tandis que d'autres définitions, par un souci sincère de classification et d'inclusion, sont tellement larges ou cataloguées (voir L'œuvre de l'art de Gérard Genette pour le catalogue des multiples modes d'immanence de l'art) qu'elles deviennent des non-définitions qui décrivent l'art comme un concept et une discipline si vastes qu'elles en font un non-domaine. Allant de la mimésis imparfaite d'une nature formalisée avec Platon7, en passant par un statut controversé d'objet de musée mort ayant autrefois servi à représenter le divin à la conscience pour Hegel ${ }^{8}$, jusqu'à devenir un objet quelconque qu'un artiste de notoriété se décide à exposer ou à « défamiliariser» avec Mutt / Duchamp 9 et (par analogie / extension) le formalisme russe de Chklovski ${ }^{10}$, fondateur de l'OPOJAZ, les définitions de cet échantillon non exhaustif mais typique sont soit trop étendues ou trop restreintes en limitant respectivement l'art à l'éthique et à la réplique imparfaite de la beauté originelle des idées, en déclarant conséquemment sa mort après la période romantique, en circonscrivant l'art à un exercice intellectuel et, finalement, en ne jugeant l'art que pour sa nouveauté ou son originalité pour éviter de parler des problèmes de la beauté. Nous n'entrerons pas dans le débat d'une définition de l'art, préférant poursuivre la discussion en utilisant le mot avec une certaine flexibilité, plasticité, souplesse et ouverture. Notre article traite d'outils conceptuels pour permettre une discussion sur l'art, sans lui donner une quelconque définition, car ces outils conceptuels peuvent également s'appliquer à des objets purement fonctionnels, comme un aqueduc, un parapluie ou un bain; un champ lexical approprié pour nous sortir du thème de la perméabilité des définitions. Nous permettons que cette mise en suspens de la définition de l'art fasse peut-être de notre méthode une grille d'interprétation heuristique et nous laissons au lecteur le soin d'en juger en espérant, bien sûr, que ce dernier soit indulgent envers nous pour cette zone laissée grise. Nous lui promettons d'aborder cette problématique de manière critique lors de la conclusion du présent article.

Laissons temporairement de côté cette mise en suspens d'une définition de l'art, et voyons dans les pages qui suivent l'opportunité de s'adonner à un

7 Platon 1963, p. 355.

8 Hegel 1995, p. 18.

9 De Duve 1989, p. 16 («monstration» de l'artiste) et p. 47 («verdict» du spectateur).

10 «Le but de l'art, c'est de donner une sensation de l'objet comme vision et non pas comme reconnaissance; le procédé de l'art est le procédé de singularisation des objets et le procédé qui consiste à obscurcir la forme, à augmenter la difficulté et la durée de la perception. L'acte de perception en art est une fin en soi et doit être prolongé; l'art est un moyen d'éprouver le devenir de l'objet, ce qui est déjà «devenu» n'importe pas pour l’art». (Chklovski 2001, p. 82). 
exercice intellectuel original et utile en se servant de certaines idées de Gould pour exemplifier une mise en application de notre approche heuristique fonctionnaliste à la technologie en musique. Pour s'attaquer au sujet, la préférence du pianiste pour le studio d'enregistrement semble être un bon point où commencer notre analyse. «I do not like playing in public so much because I always feel there are three thousand pair of eyes watching what I do rather than listening ${ }^{11}$ ", affirme Gould en décrivant son aversion pour la performance publique. Dans ce passage en particulier, le pianiste semble faire au moins deux jugements critiques: il évoque une idée pratique où la salle de concert comme médium de présentation de l'œuvre implique des restrictions physiques et des caractéristiques réceptives subjectives pour l'auditeur ainsi qu'une idée à caractère éthique sur l'écoute et le jugement du public. Commençons en analysant le premier: l'échec de la communication artiste-auditoire dans le contexte de la performance en direct.

\section{LA POSSIBILITÉ DE SE RAPPROCHER DE LA PERFECTION POUR LA DIFFUSER}

Nous traitons ici de l'idée particulière qui consiste à amputer les aspects visuel et local de la performance en direct, de ses contingences humaines et personnelles, pour permettre une pure «contemplation» de l'aspect sonore et intentionnel du musicien grâce à la manipulation technologique. À quoi pas utile référons-nous lorsque nous parlons d'une écoute "pure»? Qu'est-ce qui est typiquement contemplé lors d'une écoute phonographique? À quoi doivent être fidèles ces enregistrements? Doivent-ils être des rendus justes de la performance? Est-ce que la performance doit être plutôt un moyen de parvenir à une autre finalité? L'enregistrement doit-il être fidèle à l'intention du musicien ou doit-il être fidèle à ce que l'auditeur s'attend à entendre (l'honnêteté photographique absolue ${ }^{12}$ versus une construction élaborée)?

En supposant qu'il y ait deux types de paradigmes ou d'idéaux de l'enregistrement musical, une division méthodologique fonctionnelle synthétisée par le musicologue finnois Juha Markus Mantere ${ }^{13}$, où l'un serait phonographique (une construction assemblée en studio d'un évènement idéal) et où l'autre serait de type archivistique ou documentaire (réplique neutre d'un évènement réel et des habiletés du musicien pouvant être reproduites en live), l'idéal de Glenn Gould serait totalement du côté phonographique pour la réalisabilité in concreto de son idéal de perfection. Pour le pianiste, l'enregistrement n'est pas la réplique d'une expérience de concert, mais un procédé artistique subjectif influencé par la vision esthétique, les goûts personnels et le talent technique de ceux qui y

11 Ostwald 1997, p. 91.

12 Pour Eco, même une prise de vue télévisée en direct n'est «jamais une reproduction pure et simple de l'évènement», mais plutôt une «interprétation", car elle présente toujours un point de vue plus ou moins intentionnel. (Voir Eco 2003, p. 149). Pour Guertin, il y a déjà une ambigüité à vouloir une communication de sens parfaite. Il s'agit d'un problème de traduction qui débute aussi tôt qu'à l'étape de la lecture et de l'interprétation d'une partition. (Voir Guertin 2007, p. 173).

13 Mantere 2006, p. 147. 
œuvrent ${ }^{14}$, un processus artistique par lequel les «indésirables restrictions naturelles", de même que les «restrictions imposées à l'imagination par l'habitude de la performance publique», ont été court-circuitées ${ }^{15}$. On parle ici d'un mode de conduite / un protocole où il y aurait une finalité désirable à atteindre (un idéal). Comme cela est le cas dans la théorie utilitariste de John Stuart Mill, une éthique conséquentialiste pragmatique et hédoniste, où une finalité désirable justifie les moyens pour y parvenir ${ }^{16}$, la matrice finale est tout ce qui compte, et ce, peu importe les centaines de prises sonores nécessaires pour y parvenir; telle était exactement l'opinion de Gould, et cela même avant qu'il ne mette fin entièrement à ses concerts ${ }^{17}$. La performance devient donc le moyen d'atteindre une fin idéale réalisable concrètement par la phonographie.

\section{UNE ÉTHIQUE (OU UNE ESTHÉTIQUE ?) DE L'ENREGISTREMENT}

Étant des êtres dotés de raison, d'imagination et de langage, nous avons la possibilité d'imaginer, à partir de notre expérience, une condition meilleure que celle observée. Ces jugements sont très subjectifs et relatifs. Certains hommes observent une performance, classique par exemple, et la considèrent comme un idéal auquel il nous faut aspirer pour son réalisme ou pour n'importe quelle autre raison. Certains autres hommes observent la même performance et l'imaginent ensuite avec des caractéristiques supplémentaires qu'ils jugent meilleures (entendre la section des cordes encore plus largement, ou éliminer toutes les erreurs et même «l'humanité » en entier de la performance). Ce sont là des jugements de valeur qui sont discutables et sujets à débattre. Le point de vue de Gould correspond au deuxième type d'observateur; ce dernier utilise la performance et la technologie (indispensable) comme moyen pour parvenir à sa performance idéale: "I have not since then been able to think of the potential of music (or for that matter of my own potential as a musician) without some reference to the limitless possibilities of the broadcasting / recording medium ${ }^{18}$ ", affirme Gould avec enthousiasme. Ce point de vue fonctionnel sur la technologie est, certes, positif (dans le sens du positivisme), mais il ne nous semble pas totalement naïf, comme cela peut être le cas à différents degrés pour tout type de positivisme où il est parfois difficile de faire la distinction entre la foi en l'objet du positivisme (la foi en la technologie dans ce cas) et la raison critique. Un tel type de positivisme crédule présuppose que toute technologie est nécessairement bonne en soi en y associant une amélioration inévitable et généralisée de l'état des choses. Nous ne sommes pas entièrement en présence d'un tel cas avec Gould, car sa position est davantage réfléchie. Autrement dit, et ceci s'applique également à la doctrine utilitariste de John Stuart Mill, pour Gould la technologie est souhaitable, non pas en soi, mais en tant qu'elle peut fournir les moyens nécessaires à l'atteinte d'une finalité désirable. L'enre-

\footnotetext{
14 Michaud 2012, p. 77.

15 Gould 1966, web.

16 Mill 2002, p. 239.

17 Gould 1990, p. 287.

18 Ostwald 1997, p. 92.
} 
gistrement en studio permettant l'édition est un moyen désirable parce qu'il contribue à l'atteinte d'une finalité, elle aussi désirable, qui est différente des nombreuses performances (moyens pour atteindre la finalité) ${ }^{19}$. Quel est donc ce résultat et pourquoi est-il souhaitable?

Ce résultat final est un rendu auditif / physique d'un idéal, sans obstacles ou contingences non contrôlées: c'est un mélange son / musique, tel que désiré et imaginé par le musicien. Pour Gould, le studio était précisément l'endroit où une telle transmission de son intentionnalité était possible, car il était en mesure de jouer et de répéter jusqu'à ce que son «message musical» soit «parfait ${ }^{20} »$. D'un point de vue pratique, nous avons des doutes quant à l'existence possible d'une transmission pure et d'une réception pure, c'est-à-dire d'une transmission et d'une réception de data factuellement ${ }^{21}$ sans interprétations. Nous ne sommes pas non plus certain que Gould aille aussi loin.

Dans son ouvrage Manière de faire des mondes, Nelson Goodman, qui aborde précisément ce sujet, affirme que la musique ne se perçoit pas seulement qu'au son et que, comme tout art non descriptif, elle «fonctionne» avec des symboles qu'elle possède littéralement ou métaphoriquement. Ces symboles, qui proviennent de notre monde (notre vécu sensible), nous aident ensuite à «re-connaitre ${ }^{22}$ » la musique. Pour Goodman, la littérature, la science et la philosophie marchent également selon ce même mode de fonctionnement ${ }^{23}$. Nous croyons que le mot «fonctionnement» est particulièrement bien choisi: dans la littérature philosophique traditionnelle depuis Platon, lorsqu'on aborde la famille des termes «fonctionnels», ils se posent presque toujours en opposition à une famille de termes ontologiques / "en soi» / «le ce que c'est» des choses, car les premiers répondent aux questions «comment est-ce que c'est?» ou «comment est-ce que ça fonctionne?», qui sont considérés comme traitant $\mathrm{d}^{\prime}$ '«accidents» ou de «contingences» plutôt que d'essences des choses (presque toujours des idées). Goodman est un nominaliste ${ }^{24}$, alors la question ontologique est déjà réglée pour lui: les idées ne sont que des mots qui nous sont nécessaires pour fonctionner, sans que ce à quoi ils réfèrent coïncide avec une existence quelconque dans un autre monde. Les catégories qu'il crée (symboles littéraires et métaphoriques) ne sont pas identitaires, mais elles décrivent plutôt une relation de fonction dynamique entre l'objet traité / filtré, le sujet observant et le monde qui l'entoure. Lorsqu'il parle de la relation entre les symboles et ce qu'ils symbolisent, cette relation n'est jamais absolue ou universelle: tout cela est très relatif à l'interaction entre le phénomène musical et l'observation. Résumé en termes simples: tout input sonore vient avec un bagage d'images ou de sentiments qui n'existent pas factuellement dans la musique elle-même. Enlever la performance en direct et tout son contenu visuel n'empêche pas les gens d'être soumis à leur bagage de vécus affectant nécessairement leur

\footnotetext{
19 Ibid., p. 134.

20 Ibid., p. 188.

21 Néologismes: (1) information au sens large et (2) dans les faits.

22 Goodman 2010, p. 147-151

23 Goodman 2009, p. 79.

24 Giovannelli 2010, web.
} 
expérience sonore. Il est impossible de recevoir la musique en soi : on reçoit plutôt toujours ce que la musique est pour nous (sa fonction pour nous ou son effet sur nous). Les phénoménologies de Husserl et de Merleau-Ponty brisent également la conception selon laquelle nous observons des objets qui se tiennent devant nous: en fait, nous n'avons accès qu'à l'effet de ces objets sur nous (Husserl évite même de se prononcer sur le statut d'existence du monde). Il est, en résumé, très improbable qu'une communication pure, sonore ou pas, puisse être possible, puisque tout input d'informations ou tout phénomène est filtré et même fabriqué par le déploiement de notre conscience et son intention de signification ${ }^{25}$.

Dans ce contexte, nous pouvons nous demander s'il est réellement possible d'enregistrer une musique conformément au désir du musicien (ce qui est déjà difficile) afin de la transmettre à l'auditeur exactement comme le musicien souhaiterait qu'elle soit rendue. Une telle communication peut-elle être envisageable (ou faisable)? Nous croyons avec Goodman et Husserl qu'une transmission de stimuli allant du point " $\mathrm{A}$ » au point « $\mathrm{B}$ », une communication pure allant de l'émetteur au receveur, soit incertaine et il est très probable que Gould ait partagé cet avis, voyant en la technologie un double rôle de communication. Le premier étant technique et physique, en permettant le plus possible une écoute aussi claire qu'une lecture polyphonique d'un contrepoint sur partition alors que le second est davantage un idéal avec une visée pratique, en agissant comme un médiateur conciliateur des catégories identitaires traditionnelles en musique:

Thinking as a composer, [Gould] identified creatively with the work performed and could therefore allow himself a critical and non-servile attitude towards the score. Harnessing the undissipated intellectual power that only solitude can give, he could engage himself in a manifold enterprise as pianist, composer, writer and broadcaster: a kind of meditation in action on the concept of communication in the era of technology and of the "charitable" machine. Technology, he hoped, would be a redeemer. A sin had been committed at the end of the eighteenth century and if technology helped to put less emphasis on the notion of individual and separate identity, as well as on the hierarchical subdivisions that art seems to imply, composer, interpreter and listener should be in a state to reclaim the unity shattered by the artistic concepts of the Romantic age. ${ }^{26}$

C'est ici que nous pouvons réellement commencer à parler d'une éthique au même titre que d'une esthétique de l'enregistrement de la musique, où le discours du pianiste ne porte plus sur «ce qui est», mais sur «ce qui devrait être». La paix dans le monde, l'équité et le bonheur, la liberté et la justice pour tous, enregistrer et transmettre la musique d'une manière totalement harmonique entre l'auditeur et le musicien... que d'utopies. Doit-on pour cela stopper toute tentative pour y parvenir? Sans doute que non; la difficulté d'une quête que nous considérons comme noble pour ses effets désirables ne doit pas être un

25 Husserl 1972, p. 47. Voir aussi: Husserl 2001, p. 17.

26 Monsaingeon 1973, web. 
motif suffisant pour nous empêcher d'y aspirer. C'est là que résident les malentendus entre Gould et ceux qui le voyaient comme un excentrique maniaque du contrôle ${ }^{27}$ : il n'y a pas de folie à tendre et à aspirer à une finalité désirable quoique difficilement réalisable concrètement. Ainsi, l'enregistrement n'est sans doute pas le moyen idéal de transmettre le message musical sans interprétations ou traductions entre le phénomène phonographique et le déploiement de la conscience de celui qui le perçoit, mais il demeure tout de même le moyen pratique qui tend le plus vers cet idéal de transmission fonctionnel et non identitaire, lorsqu'il n'est pas lui-même une finalité en soi.

\section{VALEURS VÉHICULÉES}

Résumons le tout en termes fonctionnels et utilitaristes. Les catégories «composition», "performance» et «phonographie» ne sont pas des catégories identitaires, mais fonctionnelles. Chacune d'elles participe à une fonction de finalité ou de moyen dans le processus de réalisation, de diffusion et de réception d'un objet musical qui correspond à un idéal, réussi ou non, qui nous est personnel et qui coïncide avec ce que nous valorisons. Pour certains, l'idéal de cette «éthique de l'enregistrement» correspond à la réalisation de la copie carbone d'une performance sans retouches. Ils utilisent alors la phonographie comme un moyen pour rendre justice, de la manière la plus transparente possible, à leur vision de la performance en direct. Pour d'autres comme Gould, l'idéal peut correspondre (car il considère qu'une pluralité d'interprétations très différentes peuvent être valides artistiquement) ${ }^{28}$ à une vision d'une performance qui est difficilement réalisable et qui demande des aptitudes techniques et créatives non naturelles. Pour lui, la phonographie n'est pas un moyen pour imiter la performance, mais une finalité artistique à atteindre par le moyen des performances et de la technologie lors de l'enregistrement:

One cannot ever splice style-one can only splice segments which relate to a conviction about style. And whether one arrives at such a conviction pre-taping or post-taping (another of the time-transcending luxuries of recording: the post-taping reconsideration of performance), its existence is what matters, not the means by which it is effected. ${ }^{29}$

L'application de la vision fonctionnelle et utilitariste aux catégories de la composition, de la performance et de la phonographie permet d'évacuer du débat les termes «malhonnête», «tromperies» ou «mensonges ${ }^{30}$ » lorsque nous nous référons à la manipulation de la performance par le biais de la technologie dans la phonographie. Il serait d'ailleurs intéressant d'effectuer une étude ethnomusicologique / historique traitant des formes alèthurgiques (étude des formes de la vérité) du type de phonographie «documentaire», afin de mettre

27 Gould affirme avoir déjà passé trois fins de semaines complètes de 16 heures avec son équipe, seulement pour corriger la syntaxe et les maladresses verbales («um», «uh», "sort of») de ses entrevues pour l'émission The Latecomers. (Voir Payzant 1978, p. 133).

28 Monsaingeon 1973 , web.

29 Gould 1966, web.

30 Payzant 1978, p.121. 
en lumière de manière détaillée le processus par lequel ce dernier apparait à certains comme un processus qui génère un type de vérité. Nous pensons contribuer, en partie, à cet office en décortiquant les catégories des moyens et des finalités dans la création artistique, mais il y a ici un sujet au potentiel vaste en contenu, qui pourrait être exploré dans le cadre d'une thèse doctorale et qui dépasse la portée du présent article.

Il y a peut-être une sorte de tromperie lorsqu'une personne fait miroiter une fausse impression pour manipuler et satisfaire les attentes de l'auditoire qu'elle cherche à acquérir. Cette forme de tromperie doit être abordée par l'éthique traditionnelle comme une forme de mensonge et non par une théorie esthétique de l'enregistrement. Nous ne nous attaquons pas à ce premier type ici, une morale à obligations et sanctions; disons simplement que personne n'est mystifié devant la question à savoir si la tromperie est dommageable au genre humain. Pour Gould, et en ce qui concerne la question esthétique, l'atteinte d'un objet musical idéal passe par la fabrication phonographique : «I love recording because if something lovely does happen, there is a sense of permanence, and if it doesn't happen, one has a second chance to achieve an ideal ${ }^{31}{ }^{1}$. Par contre, et en ce qui concerne l'idée de la production phonographique en tant qu'art de mise en scène «malhonnête», il y a peut-être trop de ces gens qui croient encore (et / ou aiment croire) que la lutte WWE télévisée n’est pas truquée ou, mis en termes négatifs, il y a peut-être trop de ces gens qui ne veulent pas voir et apprécier la lutte comme un spectacle divertissant (World Wrestling Entertainment). Nous ne connaissons personne qui accuse le cinéma d'être un art malhonnête (ou non documentaire), mais nous entendons régulièrement certains collègues s'opposer à l'édition de performances musicales pour les fins d'un enregistrement en soutenant leurs propos par des arguments éthiques de portée universelle. Pourtant, la plupart des acteurs du grand écran, à l'exception de certains partisans du Dogme95 et du néoréalisme, reprennent constamment des scènes, qui sont ensuite mises dans des ordres qui ne correspondent pas à la série chronologique du tournage, mais qui concordent plutôt avec une chronologie idéale: il s'agit de l'ordre de l'histoire du film. À propos de cette rhétorique moraliste, John McClure (directeur de Masterworks à Columbia Records), est cité dans l'article pilier «The prospects of recording» de Glenn Gould :

Tape splicing isn't a moral question at all, any more than the number of stagehands used backstage at a play production is a moral question or the number of revisions of a book is a moral question. It's really the product that counts. The consumer's only concern should be what he hears and how he reacts to what he hears. He has a legitimate complaint only when the splicing technique actually does affect the final product, when the impact or the over-all line is damaged because of obvious inserts. ${ }^{32}$

L’enregistrement phonographique peut avoir une visée différente de la performance devant public tout comme le cinéma peut avoir un objectif différent

31 Ibid., p. 125.

32 McClure 1966, web. 
de celui du théâtre, et il nous faut aborder l'un ou l'autre d'une manière fonctionnelle, pour ce qu'il est, en fonction de sa finalité.

Un dernier avantage de l'utilisation du système de catégories fonctionnelles et utilitaires: une meilleure compréhension du débat sur la supériorité d'un genre musical par rapport à un autre (par exemple la musique classique versus la musique populaire). Une lecture appuyée par une méthodologie utilitariste appliquée à l'esthétique pourrait consister à penser que l'objectif de toute forme d'art est le plaisir, ce qui peut revenir dangereusement à limiter l'art à l'éthique, mais, à des fins d'exemple, cette lecture peut être un exercice utile pour nous aider à révéler les idéaux éthiques impliqués dans les jugements esthétiques de la vie courante. «La musique classique est beaucoup plus complexe que la musique populaire, qui ne fait que procurer du plaisir »; nous avons constamment l'occasion d'entendre des affirmations de ce genre de la part d'une grande variété d'individus provenant de domaines hétérogènes. Une affirmation comme celle-ci pose un jugement sur les types de plaisirs qu'un être humain peut ressentir. C'est pour cette raison que les épicuriens étaient traditionnellement accusés de professer une doctrine pour les animaux (les porcs spécifiquement). Ces derniers répondaient alors à leurs détracteurs que c'était plutôt eux, qui se rendaient coupables d'entretenir une mauvaise vision de l'homme en considérant que l'humain ne pouvait ressentir d'autres plaisirs que ceux d'un porc. Il nous est possible d'avoir du plaisir en lisant un livre, comme il nous est possible d'avoir du plaisir en mangeant. Il nous est possible d'avoir du plaisir en réfléchissant aux harmonies et aux paroles qui décrivent les grandes aspirations de l'Homme dans le Crucifixus de Caldara et il nous est également possible d'avoir du plaisir en dansant sur la musique de Marie-Mai, remplis du bonheur de ne pas être concentrés sur nos pensées sérieuses ou nos problèmes du moment. Rien n'empêche également qu'il soit aussi possible de ressentir du plaisir intellectuel en réfléchissant aux œuvres de Marie-Mai avec une ardeur proportionnelle ou non à la valeur de son œuvre. L'un n'exclut pas l'autre, mais certaines personnes, habituées à limiter l'art à l'éthique, ne semblent être capables d'apprécier qu'un seul de ces deux types de plaisirs. La même réflexion est applicable aux douleurs d'une manière inverse. Comme le dirait si bien un riche mercantile libéral du XIX ${ }^{\mathrm{e}}$ siècle (un exemple imagé) : « une attaque publique à l'honneur ne ferait qu'effleurer un sauvage, tandis que le même assaut serait suffisant pour tuer et enterrer un homme civilisé». Cette conception n'exclut pas non plus la pertinence ou l'existence de musiques pouvant être décrites comme désagréables (musique de films d'horreur, musique gore, etc.), car celles-ci peuvent être instrumentales à d'autres types de plaisirs comme la catharsis (purgation de pulsions de mort), l'humour ou le plaisir de mettre les autres dans l'inconfort, quoique ce dernier ne serait peut-être pas recommandé par la moralité utilitariste avant de passer par une bonne discussion mettant en vedette un calcul des plaisirs et des douleurs encourus.

Lorsque nous débattons de la supériorité d'un genre musical sur un autre, nous sommes plutôt en train de décrire quelle est notre vision idéale de l'homme. Est-ce que le corps est inférieur à l'esprit? Peut-on rationnellement rejeter l'un ou l'autre? Est-ce que ces deux sont séparables? Y a-t-il un plaisir supérieur 
à un autre? Mill, le père de l'Utilitarisme, dirait que oui: un plaisir de l'esprit est préférable à un plaisir corporel, lorsque comparés en quantités égales. Les expériences et les témoignages de ceux qui ont vécu les deux types de bonheurs en seraient la preuve. Nous sommes, quant à nous, très hésitants à déclarer la primauté de l'un sur l'autre de même qu'à associer l'art à l'éthique en le limitant au plaisir. Malgré la tentation de voir dans cette association "plaisirart» une occasion d'y construire un système esthétique logiquement solide et presqu'universel, où la fonction artistique est hédoniste, nous y voyons le risque d'exclure certains arts qui souhaitent non seulement ne pas être associés au plaisir, mais qui, en plus, ne désirent avoir aucune signification intentionnelle. Nous avons une façon très particulière de vivre l'art (subjective et orientée, mais vécue comme objective, nous pourrions convenir avec Kant et Jauss) ${ }^{33}$, mais aucune de ces deux polarités incompatibles ne nous satisfait tout à fait, à elle seule, intellectuellement. Gardons seulement le plaisir, qui est toujours désirable peu importe son type lorsqu'il n'entraîne pas une douleur qui lui est supérieure, en dehors du débat pour justifier une hiérarchisation des arts.

\section{L'ENREGISTREMENT DANS L'APPRENTISSAGE: SON RÔLE PARRÊSIASTE}

L'enregistrement fournit au musicien des avantages didactiques indiscutables, surtout lorsqu'il est perfectionniste et autodidacte, en rajoutant une couche supplémentaire d'art sur l'art: en pouvant s'écouter le nombre de fois qu'il le souhaite après s'être exercé, le musicien peut dédier toute son attention à l'écoute et à l'art de rendre cette écoute pour une réception quelconque visée, au lieu de partager son attention entre cette écoute critique et l'expérience de sa performance. En s'observant jouer après coup, il est confronté à soi-même d'une manière plus détachée que lorsqu'il joue simultanément de son instrument, n'ayant pas à distinguer ce qu'il entend de ce qu'il ressent, émotionnellement parlant, lors de la performance ${ }^{34}$. Les aspects de son jeu (de dynamique, de tempo, d'articulation, etc.) lui apparaissent plus froidement que les critiques constructives de ses pairs : les ornements, les métaphores, les politesses et le style linguistique sont court-circuités dans l'enregistrement. Notons que nous exposons certains avantages de ce discours de vérité métalinguistique, sans toutefois défendre l'élimination des conseils d'un pair.

En ce qui concerne Gould, ayant appris très jeune à se servir d'une enregistreuse à ruban, il ne s'en sépara jamais, et alla même jusqu'à affirmer à un ami que cette machine à vérité était le meilleur de tous les enseignants et que sans elle il serait "perdu 35 ». Le mot "perdu» est très puissant: outre son rôle didactique, l'enregistrement permet une connaissance de soi-même (il s'agit peut-être d'une des fonctions de l'art?) ou, plus spécifiquement, une vérité sur sa propre performance, sans laquelle l'interprète est "perdu» / il ne se reconnaît pas lui-même, car il est très difficile de s'entendre jouer avec une lucidité

\footnotetext{
33 Jauss 2007, p 75-76.

34 Ostwald 1997, p. 89.

35 Ostwald 1997, p. 89.
} 
détachée lors de la performance. Cette dernière affirmation nous rappelle les paroles du champion de la connaissance de soi, Socrate (le parrêsiaste [celui qui dit la vérité] par excellence selon Michel Foucault), dès les premières lignes de son apologie par Platon:

Je ne sais, Athéniens, quelle impression mes accusateurs ont fait sur vous. Pour moi, en les entendant, peu s'en est fallu que je me méconnusse moimême tant ils ont parlé d'une manière persuasive; et cependant, à parler franchement, ils n'ont pas dit un mot qui soit véritable. Mais, parmi tous les mensonges qu'ils ont débités, ce qui m’a le plus surpris, c'est lorsqu'ils vous ont recommandé de vous bien tenir en garde contre mon éloquence... j'avoue alors que je suis un habile orateur, mais non pas à leur manière; car encore une fois, ils n'ont pas dit un mot qui soit véritable; et de ma bouche, vous entendrez la vérité tout entière, non pas, il est vrai, Athéniens, dans les discours étudies, comme ceux de mes adversaires, et brillants de tous ces artifices du langage... ${ }^{36}$

Nous lisons ici l'importance du souci de vérité sur soi-même, une vérité en l'absence de laquelle nous sommes "perdus» ou nous ne nous reconnaissons plus nous-mêmes. On y discerne également l'importance d'une communication par un langage "sans artifices», une condition alèthurgique foucaldienne ${ }^{37}$, pour qu'une description de soi puisse être perçue comme vraie. Il s'agit effectivement de concepts fondamentaux dans la notion de parrêsia développée par Michel Foucault durant les dernières années de sa vie et d'enseignement au Collège de France (1980-1984) où il s'intéressait aux formes de présentation de la vérité et du dire vrai (études des formes alèthurgiques / études des formes de la vérité). Foucault analyse les structures des discours du dire vrai ou les types d'actes par lesquels l'individu qui dit la vérité est reconnu par les autres comme un sujet tenant un discours de vérité ${ }^{3}$. Sous quelles formes se présente à ses yeux et aux yeux des autres celui qui tient le discours du dire vrai?

En Occident, la tradition du principe selon lequel il faut dire le vrai sur soimême est extrêmement importante (Foucault débute son analyse avec les Pythagoriciens). Le parrêsiaste est celui qui dit tout / sans rien dissimuler. Nous faisons ici référence à une valeur positive de la parrêsia, qui consiste à dire la vérité sans en cacher une partie et sans figures de style, et non à dire absolument tout ce qui traverse notre pensée ou ce qui peut desservir notre propre cause. Le parrêsiaste a un rôle normatif risqué qui diffère de celui de l'enseignement, où il n'y a généralement aucun risque, sauf peut-être lorsqu'il remet en cause les pratiques établies. Historiquement, la parrêsia se pratique entre deux hommes ou entre un homme et une foule, où un interlocuteur est nécessaire pour connaitre la vérité sur soi. Ces pratiques du dire vrai sur soi-même se sont transférées d'une époque à une autre en passant, en ordre (selon Foucault), par les pythagoriciens, Socrate, le mouvement cynique, les dominicains, l'institution de l'Église catholique, le discours psychologique et son implication dans

\footnotetext{
36 Platon [17a] à [17c], web.

37 Rojas 2012, p. 7.

38 Foucault 1984, web.
} 
les divers systèmes judiciaires ${ }^{39}$... Certains duos sont assez célèbres comme le prêtre et le pénitent, le psychanalyste et son client, le bourreau et le supplicié, l'accusé et le jury... Assistons-nous à un nouveau type de duo parrêsiastique, moins épique, plus accessible et moins risqué, soit celui du musicien interprète et de l'outil d'enregistrement sonore? L'enregistrement occuperait-il une fonction alèthurgique similaire envers le musicien et sa propre performance? L'enregistrement dévoile-t-il les imperfections de ses propres performances? Si oui, provoque-t-il une invitation à la réforme de celles-ci? De manière générale, l'art, incluant l'enregistrement, pourrait-il être un exercice de style permettant de se connaitre soi-même?

L'enregistrement peut être similaire aux pratiques historiques mentionnées plus haut, quoiqu'il y ait une différence de degrés par rapport à la pratique du dire vrai entre hommes. La captation d'une performance s'apparente davantage à des exercices spirituels comme l'entretien d'un journal: écrire ses rêves, écrire ses comptes rendus de lecture, tenir un journal intime, écrire dans un blogue, etc. Il s'agit de pratiques qui peuvent avoir comme fin intentionnelle ou non de mener à la connaissance de soi, à un examen de conscience et à la gouvernance de soi-même. Pour Gould, l'enregistrement sonore semble certainement tenir un rôle de moyen pour parvenir à la connaissance intime de ses performances et de leurs imperfections. Tout comme le parrêsiaste joue un rôle essentiel dans la connaissance de la vérité sur soi, l'enregistrement permet au musicien de connaitre la vérité sur sa performance. Il peut sans doute y avoir un aspect douloureux et risqué dans une révélation non préparée de soi ${ }^{40}$, surtout lorsque celle-ci doit mener à une réforme qui nous sort de notre confort. Y a-t-il un risque de nier cette vérité en attribuant le blâme au médium plutôt qu'au sujet de l'enregistrement?

\section{L'ASPECT MOTIVATIONNEL DU DIRE VRAI SUR SOI-MÊME}

Une caractéristique essentielle de la parrêsia, et de la fonction du dire vrai de l'enregistrement, est la finalité motivationnelle que ces pratiques ont sur ceux auxquels elles s'adressent. Foucault présente la parrêsia, comme un discours vrai sur soi-même, mais qui se traduit en un appel à la réforme de soi, rendue possible par la prise d'une distance critique par rapport à soi-même. Autrement dit, la pratique de la parrêsia nous dévoile des imperfections à propos de nous-mêmes, afin que nous puissions opérer un changement bénéfique (selon ce que nous considérons subjectivement être bon et désirable) pour mieux nous gouverner nous-mêmes. Il est possible d'objecter à cette idée la traditionnelle acrasie d'Aristote qui consiste à agir contre notre connaissance lucide et vérifiée des faits. Pourquoi est-ce qu'un enregistrement de notre performance pourrait avoir la fonction de nous influencer à agir pour réformer les imperfections de celle-ci? Il arrive souvent que l'homme ne tire pas de leçons de l'histoire. Il est également clair que l'homme agit régulièrement de manière lucide, contre ses propres intérêts. Comment la connaissance de nos imperfections

39 Idem.

40 Foucault 2004, p. 847. 
musicales performatives pourrait-elle avoir une quelconque influence motivationnelle sur nos actions? Pourquoi pouvons-nous comparer l'enregistrement à la parrêsia, à sa fonction de vérité et d'appel à la réforme de soi?

Pour répondre à ce problème, nous pouvons nous demander «qu'est-ce qui précède quoi ?». Plaçons la parrêsia et le souci de la vérité sur soi-même en termes chronologiques. La parrêsia ne nous apprend pas que nous avons des imperfections. Le désir de connaitre la vérité sur soi-même est un souci qui implique déjà notre connaissance du fait que nous soyons imparfaits. Ce souci précède le recours aux exercices spirituels du dire vrai sur soi et du dire vrai sur sa propre performance musicale. Gould était un homme compétent et critique de lui-même en raison de ses standards très élevés; il était alors bien placé pour savoir qu'il y avait des imperfections dans ses propres performances avant même de les enregistrer. Le pianiste était donc déjà, au départ, enclin à résoudre et à réformer ces imperfections; la motivation d'action est déjà contenue dans ce souci de perfection et de vérité qui est véhiculé et alimenté par nos propres valeurs et idéaux. Là où la parrêsia et l'enregistrement interviennent, c'est dans la spécification et la désignation des imperfections particulières, en tant qu'ils sont des moyens pour aspirer à une finalité de gouvernance de soi. Ultimement, en se rapprochant de plus en plus de cette finalité par le moyen de l'enregistrement, le calibre des attentes musicales, les libertés et le plaisir du musicien sont augmentés:

Lorsque je commençai à faire des enregistrements, je n'espérais rien de plus que de faire en sorte que mes interprétations en public soient aussi bonnes que l'enregistrement équivalent de la même œuvre, pour peu bien sûr que je l'aie déjà enregistrée, ce qui était d'ailleurs souvent le cas: on triche lorsqu'on donne des concerts; on n'explore guère de nouveaux répertoires, on se contente de rejouer les mêmes vieux morceaux fatigués que l'on essaie devant son public d'enregistrement aussi bien que devant son public public. On fait tout pour s'en tirer avec le moins de travail possible et on rejoue presque toujours tout à peu près de la même manière. L'absence d'imagination règne. $4^{11}$

Gould décrit bien ici l'élévation de ses standards de concert (obtenir les mêmes résultats que ses enregistrements en studio) tout en apportant une perspective opposée à celles dont nous sommes habitués d'entendre parler, en mentionnant l'existence de ce qu'il considère comme une paresse imaginative dans le milieu de la performance.

\section{SYNTHÈSE}

Pour Gould, le meilleur moyen pratique de se rapprocher d'un idéal de perfection, voire de supranaturalité, et de réception pour cette perfection, est de combiner plusieurs performances sur un enregistrement afin d'en fabriquer une seule qui s'apparente le plus possible à la finalité valorisée du musicien. De plus, le processus de production phonographique offre au musicien perfectionniste

41 Gould 1986, p. 164. 
un regard pouvant être considéré comme "vrai» sur son propre jeu. Cette écoute sans artifices langagiers est motivée et justifiée par un souci de connaître la vérité à propos de ses propres performances pour y apporter une réforme pratique. L'enregistrement de nombreuses performances est alors un moyen pour identifier les imperfections de manière spécifique afin de les prendre en charge (par le moyen de l'édition phonographique ou par l'enregistrement répété) pour parvenir in concreto à un résultat final se rapprochant d'un idéal valorisé, parfois surhumain dans le cas de Gould.

En une phrase: lorsque nous appliquons les catégories fonctionnelles / utilitaristes et la sphère de la gouvernance de soi-même à la pratique musicale de Glenn Gould, la phonographie apparait comme une fin visée (fabrication concrète se rapprochant d'un standard idéal de perfection) alors que les performances et l'édition phonographique se distinguent comme des moyens pour aspirer à la finalité valorisée par le musicien.

Nous pouvons alors comprendre pourquoi le pianiste a cessé les concerts devant public en 1964. Ce n'était certainement pas le moyen le plus optimal pour arriver à ses fins. En fait, ce moyen par lequel il est difficile de communiquer la musique est un sacrifice inutile pour le musicien: la mise en valeur du fait que l'interprétation du pianiste soit difficile à accomplir dans une situation en direct où le stress entre dans l'équation de la réussite de la performance est une pratique compétitive cruelle, voire non éthique. "The whole business about asking people to test them-selves in situations which have no need for their particular exertions is wrong as well as pointless and cruel $^{42}$ », affirme-t-il dans une entrevue. Pour un homme qui nous semble utilitariste et fonctionnel dans sa mise en pratique musicale, qui tend vers des idéaux de perfection, il n'est absolument pas étonnant que ce principe trouve sa résonance dans la pensée de John Stuart Mill:

The utilitarian morality does recognise in human beings the power of sacrificing their own greatest good for the good of others. It only refuses to admit that the sacrifice is itself a good. A sacrifice which does not increase, or tend to increase, the sum of total happiness, it considers as wasted. 43

Pour une personne utilitariste, la fin ultime visée est le bonheur, et si un sacrifice ne tend pas à aider à atteindre cette fin, ou s'il est évitable, alors le sacrifice est inutile. Pour Gould, l'objet musical final (une vision de la perfection) doit être transmis de la manière la plus optimale, de la performance à l'écoute. La performance en direct, qui ne permet pas d'atteindre cette finalité, qui est évitable et qui est même cruelle, est alors simplement perçue comme un sacrifice inutile: la primauté de l'enregistrement en studio comme moyen par excellence de concrétiser un idéal de manière sensible est une évidence pragmatique et non, comme l'affirment certains ${ }^{44}$ qui font de Gould un formaliste, un borgésien et un platonicien (des penseurs pour qui l'accès privilégié à la réalité n'est

\footnotetext{
42 Gould 1990, p. 451.

43 Mill 2002, p. 249.

44 Guertin 2007, p. 189.
} 
pas d'ordre sensible), la recherche d'une entité transcendantale qui préexiste à la concrétisation sensible de l'œuvre artistique. Il ne s'agit peut-être que d'une guerre de mots ou une différence de degrés dans la méthodologie de l'analyse, car les résultats, eux, restent les mêmes: il s'agit d'un homme pragmatique qui a des idées. Certaines de ces idées, davantage prophétiques, ne se sont toujours pas réalisées, comme sa prédiction, au milieu des années 1960, de la mort du concert par exemple: "Tchaikovsky concerts would be an absurdity to me, in the year $1999^{45}$. Il est intéressant de noter qu'il n'imaginait pas particulièrement une croissance d'un sentiment apathique pour la salle de concert, mais plutôt un jeu de pouvoirs où l'auditeur, ayant gouté aux jouissives puissances des choix «interprétatifs» offerts par la phonographie à la maison (le choix de l'équipement, le contrôle des égaliseurs ainsi que la disposition des caisses), en demanderait toujours plus. Gould concevait qu'il y aurait une demande pour un médium où l'auditeur pourrait avoir le choix de combiner, selon ses propres goûts, différentes sections d'une pièce interprétée de manières diverses pour se l'approprier jusqu'à obtenir pleine satisfaction. Dans cette perspective, le compositeur de l'avenir devait alors nécessairement penser à ces nouvelles catégories performatives et réceptives dès les premières étapes de la composition.

À notre avis, il est peu probable que la salle de concert disparaisse complètement un jour: appliquons les catégories des fins et des moyens à celle-ci et il est évident que la performance en direct a une fonction distincte de l'enregistrement en studio. Ceux qui souhaitent expérimenter la salle de concert comme Richard Morh, directeur musical de RCA Red Seal Recordings, y assistent probablement pour l'évènement en plus de la musique, une perspective peut-être moins rigide rationnellement que le point de vue de Gould, une sorte de présence qui est plus difficile à capter dans une production phonographique :

It may be my imagination, but I sometimes think a live performance does have more electricity, more excitement. There are more mistakes, of course, but if the artist is really in the vein, it can be more authentic, more vital. Many musicians freeze up in the recording studio as soon as the red light goes on. ${ }^{4}$

Les autres auditeurs «puristes» du son, situés dans des sections acoustiques défavorables de la salle, sont déçus et n'y retournent pas. Quant à l'idée d'un médium permettant de combiner plusieurs types d'interprétations, elle nous parait plausible et même tangible dans un avenir rapproché, quoiqu'il soit peu probable que l'intérêt pour une telle possibilité dépasse les frontières d'un petit groupe de chercheurs, de mélomanes ou d'audiophiles. Certaines personnes, même très compétentes, n'y verraient tout simplement pas d'intérêt:

Burton: I want to hear Klemperer's Beethoven, not do my own Beethoven.

Gould: Why not? Are you afraid of your own Beethoven?

45 Gould 1966, CBC.

46 Gould Avril 1966, web. 
Burton: I think Klemperer's got more experience with that.

Gould: But if your own Beethoven were more satisfying to you, why would you not want to indulge it?

Burton: I can't see why it would ever be more satisfying... ${ }^{47}$

En ce qui concerne l'idée de penser à la réception, voire d'être influencé par la technologie du son dès les premières étapes de la composition et vice versa (par exemple, l'idée d'un déterminisme technologique où les médiums d'écoute déterminent les gouts musicaux $)^{48}$, il serait étonnant que cela n'ait pas toujours été le cas ${ }^{49}$, quoique son application à la technologie ne soit que récente, dans un degré plus «rudimentaire» que celui imaginé par Gould. Par exemple, un compositeur romantique ayant l'habitude de se produire dans une salle exagérant les graves, où les temps de déclin et de relâchement sont allongés, pourrait rationnellement avoir le réflexe de prioriser des passages en diminuendo ou pizzicato pour les instruments graves, afin de contrer le fâcheux problème. En ce qui concerne l'enregistrement, depuis le milieu des années 1980, et plus particulièrement depuis le début des années 2000, il est courant de faire des mix et des matrices ayant un niveau de sonie élevé ainsi que des dynamiques adaptées aux médiums auxquels les chansons sont le plus souvent destinées (comme la radio et les écouteurs ear buds) $)^{5}$. Le plus souvent, l'aspect technique de ces choix (que toutes les pistes d'un mix puissent être audibles dans des milieux bruyants) a un effet stylistique, qui, par la force de l'habitude, cesse d'être un effet secondaire et contingent, mais devient une esthétique recherchée et indépendante en droit. Il est tout de même assez rare, par contre, que l'aspect interprétatif soit influencé par une prise de conscience du résultat ultime à la matrice finale, en dehors des prérequis habituels des styles respectifs des chansons. Il s'agit néanmoins d'une formule audionumérique fonctionnaliste que nous jugeons très prometteuse depuis quelques années ${ }^{51}$, où nous défendons la pertinence de travailler avec la compression dès les premières étapes de la production phonographique; de composer, mixer et matricer en même temps, une méthode qui consiste à voir le processus de création comme un tout organique au service de sa finalité (la matrice finale), afin de permettre un contrôle maximal au musicien sur la compression, ses artéfacts (distorsions, perte de gamme dynamique et déséquilibre de la scène virtuelle d'un mix) ${ }^{52}$, et plus globalement, sur son œuvre musicale comme produit final.

47 Gould 1966, CBC.

48 Ashby 2010, p. 165.

49 Nous pouvons par exemple penser à l'influence qu'a pu avoir l'acoustique des basiliques sur la définition stylistique des premiers chants chrétiens à l'ère romaine. «Sung words carried better and more clearly through the large, resonant space than did spoken words» (Burkholder, Grout et Palisca 2006, p. 26).

50 Pour une description de l'escalade des dernières décennies dans les pratiques réduisant la gamme dynamique pour obtenir une sonie plus forte (loudness war), voir Katz Bob 2002, p. 271-278. Pour une description plus détaillée des facteurs perceptifs de la sonie (jugement subjectif du niveau sonore d'une pièce), voir Brixen 2011, p. 52.

51 Chiriac 2014, $128 \mathrm{p}$.

52 Moulton 2002, p. 236. 


\section{MALAISES DU FONCTIONNALISME EN ART}

Comme mentionné au début de cet article, des problèmes demeurent non résolus lorsque, sans définir l'art, on l'aborde avec des catégories fonctionnelles applicables à n'importe quel objet ayant une fonction, artistique ou non. Ces problèmes sont reliés à une régression ontologique impossible à résoudre dans presque tout système esthétique à prétention universelle, sans jugements arbitraires, et ce, même lorsqu'on essaie d'éviter de définir l'art en employant d'autres termes sophistiqués comme "beauté», "fonction esthétique», "articité53 d'un objet esthétique» et «style».

Supposons que l'on veuille discuter de la valeur d'une œuvre: dans un système de pensée fonctionnaliste, la beauté ou la perfection d'une chose pourrait être jugée en fonction du degré d'accomplissement de la fin circonstancielle (la finalité souhaitée par son créateur) qui lui est propre, afin d'éviter une des réductions courantes du terme (beauté = moralité / nature / idéal transcendantal). Dans cette logique, on peut employer le terme «beauté» même pour des objets non artistiques, naturels ou fonctionnels. Par exemple, une termitière peut être jugée belle puisqu'elle remplit sa fonction d'abriter de larges colonies sophistiquées de termites. La complexité du réseau des tunnels et du système d'aération de l'ensemble est jugée belle et désirable en tant qu'elle est un moyen de parvenir à la finalité plus large qui consiste à abriter la colonie de termites. L'œil et l'oreille de l'homme en santé sont magnifiques, puisqu'ils accomplissent gracieusement l'office de la vision et de l'ouie avec un degré de finesse et de subtilités dynamiques inouïes, qui nécessitent habituellement des changements constants dans le calibrage de nos appareils de mesure de la lumière et du son les plus sophistiqués, simplement pour pouvoir couvrir la même gamme spectrale et dynamique que ces organes conçus naturellement. Jusqu'ici, il n'y a pas trop de malaise à affirmer que les œuvres de la génétique et des termites peuvent être considérées comme belles pour leur fonctionnalité, quoiqu'il y ait clairement une difficulté quant à savoir si on peut légitimement les qualifier comme ayant vaguement (on refuse toujours de définir l'art) un caractère esthétique sans le prérequis minimum énoncé dans la définition provisoire de l'art de Gérard Genette: soit la présence d'une intention humaine de produire un artéfact ayant une fonction esthétique ${ }^{54}$.

On peut imaginer toutes sortes d'exemples apportant des exceptions et des dilemmes arbitraires (un jugement qui ne repose pas sur des catégories universelles, mais sur des circonstances aléatoires) à ces prérequis pourtant extrêmement élémentaires. Imaginons le joli chant d'un oiseau, qui ne se qualifie pas comme objet artistique selon les critères humain et intentionnel de Genette, imité à la perfection par une femme. Nous pouvons faire entendre les deux interprétations à un public expert et non expert à l'aide d'un hautparleur, sans une mise en contexte préalable sur l'origine des deux sons. Il apparaîtrait alors à quiconque qu'il serait arbitraire de désigner une interprétation comme esthétique ou artistique sans accorder le même statut à l'autre, si l'idée même

53 Néologisme: statut d'objet d'art.

54 Genette 2010, p. 13. 
d'en designer un comme artistique n'apparaît pas extravagante en premier lieu. Avec une mise en contexte, telle une légende à côté d'un tableau dans une galerie d'art, le cas devient différent, mais nous pourrions nous demander si l'articité de l'œuvre vient de son explication ou de sa démarche, puisque sans elle, l'objet est perçu comme familier ou non artistique. Il nous semble assez difficile de rejeter totalement ces outils de contextualisation, car ils nous permettent, par l'accroissement de la connaissance de l'œuvre que nous observons, d'être intellectuellement mieux équipés pour l'apprécier. Gould ne semble pas particulièrement attaché à cette contextualisation, du moins en tant que considération servant à la détermination de la valeur d'une œuvre d'art, lorsqu'il analyse le cas du brillant faussaire Van Meegeren, son «héros privé », qui réussit à duper les meilleurs spécialistes de son temps en faisant passer ses œuvres pour celles de maitres Hollandais le précédant ${ }^{55}$. Le pianiste reproche ainsi aux connaisseurs de formuler leur appréciation esthétique ainsi que leur évaluation monétaire des œuvres en ne considérant que la démarche artistique ainsi que les catégories identitaires étant rattachées aux œuvres analysées (le contexte de la réalisation ou la période historique dans laquelle l'œuvre s'inscrit ainsi que le nom et la réputation de son artisan). Selon Gould, de telles considérations favorisent un climat de conservatisme en plus de divertir l'attention sur des éléments contingents à une analyse juste :

The determination of the value of a work of art according to the information available about it is a most delinquent form of aesthetic appraisal. Indeed, it strives to avoid appraisal on any ground other than that which has been prepared by previous appraisals. The moment this tyranny of appraisaldom is confronted by confused chronological evidence, the moment it is denied a predetermined historical niche in which to lock the object of its analysis, it becomes unserviceable and its proponents hysterical. The furor that greeted Van Meegeren's conflicting testimony, his alternate roles of hero and villain, scholar and fraud, decisively demonstrated the degree to which an aesthetic response was genuinely involved..$^{6}$

Dans l'extrait précédent, les critiques décrits par Gould apparaissent en opposition directe avec l'approche fonctionnaliste, pour qui le résultat ou la finalité justifie le moyen utilisé et non le contraire, et c'est précisément ce même point qui cause l'incompréhension entre Gould et les défenseurs de l'enregistrement des performances publiques, car ils n'ont pas les mêmes objectifs et les mêmes moyens pour y parvenir:

Most aesthetic analysis confines itself to background description and avoids the foreground manipulation of the object being analyzed. And this fact alone, discarding the idle propaganda of the public-relations machines, accounts for the endorsement of the recorded public event. Indirectly, the real object of this endorsement is a hopelessly outmoded system of aesthetic analysis - a system incapable of a contribution in the

55 Gould avril 1966, web.

56 Gould avril 1966, web. 
electronic age but the only system for which most spokesmen of the arts are trained. 57

Encore une fois, il nous apparaît plutôt difficile d'aller aussi loin dans la pensée (cohérente) de Gould, mais d'un autre côté, si on ne le fait pas, si nous acceptons que le statut d'articité d'une œuvre ne dépende que du «background» à côté ou factuellement non inclus dans celle-ci, de ses idées et, bref, de sa démarche intellectuelle mise en contexte, peut-on refuser le statut d'articité à un ouvrage intellectuel (comme un exposé mathématique ou ce texte même) sans se rendre coupable d'une quelconque forme de jugement discriminatoire ou arbitraire? Avec l'analyse historique d'artistes comme Duchamp, Warhol et d'autres du mouvement Dada, on peut être tentés avec Genette d'y ajouter une condition de défamiliarisation par un artiste ayant un statut reconnu, ce qui rend tout objet fonctionnel (n'ayant pas une intention esthétique) un candidat potentiel au statut d'œuvre d'art.

En acceptant cela, on retombe dans la problématique de la définition si large de l'art, qu'on en fait une non-définition, car il faudra également accepter celui, qui, un jour, voudra se réclamer comme l'artiste d'une montagne ou d'une station de service. Pourquoi, et ne s'agit-il pas d'une pente fatale? Non, parce qu'on ne pourra pas lui refuser cette défamiliarisation comme exercice artistique sans faire preuve d'un jugement arbitraire. À notre avis, et sans rejeter le statut d'œuvres d'art des artistes de la défamiliarisation, une définition si large ne remplit plus sa fonction de « définir»; elle est plutôt une perte de temps: elle n'apporte pas un savoir positif qui puisse nous renseigner sur l'art et elle requiert trop d'exceptions capricieuses, tels les derniers systèmes cosmologiques géocentriques. La définition devient alors plus difficile à connaître que l'œuvre qu'elle cherche à décrire en plus de frôler la régression à infini:

- Qu'est-ce qu'une œuvre d'art?

- Il s'agit d'un objet défamiliarisé par un artiste.

- Qu'est-ce qu'un objet défamiliarisé par un artiste?

- Il s'agit d'un objet familier complexifié par un artiste...

De plus, nous aboutissons malheureusement à une tautologie lorsque nous associons la beauté d'un objet fonctionnel au style ou au bon goût, et l'embarras est d'autant plus profond quand vient le temps de définir universellement ces termes sans faire preuve d'un jugement arbitraire. Ainsi, une définition de l'art, dans un canon esthétique et philosophique universel, est moins utile qu'une abstention de définir l'art, mais sans elle, nous sommes contraints d'être non spécifiques par rigueur intellectuelle à propos des termes «beauté», «fonction esthétique», "articité d'un objet esthétique», «style» et "bon goût», ce qui est très peu satisfaisant intellectuellement comme point de conclusion, mais plutôt stimulant comme point de départ pour faire progresser une discussion non arbitraire en arts.

Pour clore l'article sur une réflexion à valeur positive, nous souhaitons offrir quelques avenues non nihilistes aux chercheurs et aux créateurs, qui ne sauraient plus, respectivement, [1] de quelle manière il est possible de conduire 
une étude esthétique de l'art (sans se contraindre à analyser les œuvres individuellement ou historiquement) et [2] de quelle manière il est possible d'être original alors qu'il peut nous sembler que presque toutes les limites à la liberté artistique ont été abolies par les artistes nous ayant précédé.

[1] Il nous est difficile d'imaginer un canon philosophie esthétique à prétention universelle qui n'aurait pas une théorie construite à partir de l'axiome négatif de l'impossibilité d'offrir une définition universelle satisfaisante de l'art: ça ne serait ni pragmatique, ni théoriquement solide, ni en connexion avec la pratique artistique actuelle. Si un tel canon philosophique est abordé d'un point de vue fonctionnaliste, il faut s'attendre à se heurter aux problèmes ontologiques mentionnés précédemment ainsi qu'à laisser les questions leur étant relatives sans réponses. Traiter de la question ontologique avec une réponse nominaliste pour permettre une discussion non arbitraire en esthétique, comme nous l'avons fait, tend à relativiser et à déconstruire toute théorie esthétique. Un apport de la linguistique et de la traduction pour aborder le style comme un langage et synthétiser un processus de génération de signification en arts qui soit cognitif et fondé biologiquement pourrait possiblement avoir un rayonnement intellectuel plus intéressant pour l'ébauche d'une théorie esthétique à prétention universelle sans nécessiter un positionnement ontologique idéaliste. Nous demeurons cependant silencieux quant aux moyens précis pour atteindre cette description esthétique, car il s'agit de domaines auxquels nous n'avons été exposé que de façon dilettante et car nous soupçonnons que des efforts cartésiens de doute ainsi que des efforts bibliographiques de moines soient nécessaires pour l'ébauche d'une telle théorie, sans même jamais être à l'abri d'une sérieuse critique méthodologique.

[2] Que fait-on lorsqu'il ne reste plus de règles à enfreindre et que toute approche artistique devient relative? On se crée des règles subjectives / arbitraires universellement parlant, mais qu'on justifie subjectivement, pour se fonder une esthétique singulière, un cercle ou une école de pensée théorétique, avec des amis et collègues artistes, qui nous est propre et qui, une fois établie, aura fait avancer le dialogue artistique et sera elle aussi rejetée par les générations futures d'artistes. Une fois que le créateur-chercheur (l'artiste qui a réfléchi à un programme esthétique) excelle dans ce système fabriqué de toutes pièces, comme le système dodécaphonique (génial et tout-à-fait arbitraire) développé par Hauer et Schoenberg, son œuvre est considérée comme valide pour luimême et originale sur le moment. Il est vrai que le fait de se fixer à soi-même des règles subjectives peut d'une certaine manière aller à l'encontre du propos du présent exposé. Il demeure néanmoins que la plupart des écoles esthétiques partagent un point commun avec notre article: le désir d'enrichir le dialogue à propos des arts. En ce qui nous concerne, nous avons souhaité, à l'aide de catégories logiques fonctionnalistes et utilitaristes, exposer les mécanismes arbitraires de ces jugements en esthétique dans le but de faire progresser la discussion. 


\section{RÉFÉRENCES}

Ashby, Arved. 2010. Absolute Music, Mechanical Reproduction. Berkeley : University of California Press.

Brixen Bogh, Eddy. 2011. Audio Metering, Measurements, standards and practice, $2^{\mathrm{e}}$ edition. Oxford : Focal Press.

Burkholder, J. Peter, D. J. Grout et C.V. Palisca. 2006. A history of western music, $7^{\mathrm{e}}$ edition. New York : Norton \& Company.

Chiriac, Dragos. 2014. "Le contrôle de la compression, de la prise de son au matriçage». Mémoire de maîtrise, Université Laval, Québec.

Chklovski, Viktor. 2001. "L'art comme procédé». Théorie de la littérature: Textes des Formalistes russes, sous la dir. de Tzvetan Todorov, 75-97. Paris: Seuil.

De Duve, Thierry. 1989. Résonances du readymade : Duchamp entre avant-garde et tradition. Paris: J. Chambon.

Eco, Umberto. 2003. L'œuvre ouverte. Paris: Seuil.

Foucault Michel. 2004. Philosophie Anthologie: Les techniques de soi. Paris: Gallimard, Folio essais.

—. Le courage de la vérité, archive numérique du séminaire donné au Collège de France, $1^{\mathrm{e}}$ et 8 février 1984, http://michel-foucault-archives.org/ ?Cours-au-college-de-France-1984.

Genette, Gérard. 2010. L'œuvre de l'art. Paris: Éd. Du Seuil

Giovannelli, Alessandro. 2010. Goodman's Aesthetics, The Standford Encyclopedia of Philosophy, edition été, http://plato.stanford.edu/entries/goodman -aesthetics/

Goodman, Nelson. 2009. L'art en théorie et en action. Paris: Gallimard, Folio essais.

_. 2010. Manières de faire des mondes. Paris: Gallimard, Folio essais.

Gould, Glenn. 1990. The Glenn Gould Reader, sous la dir. De Tim Page. New York: Vintage Books.

—.1986. Non, je ne suis pas du tout un excentrique, montage par Bruno Monsaingeon, France.

—.1966. Conversations with Glenn Gould (Bach \& Beethoven), entrevue réalisée par Humphrey Burton, CBC.

- 1966. "The prospects of recording». High Fidelity, vol. 16, no. 4, Avril, https://www.collectionscanada.gc.ca/glenngould/o28010-4020.01-e.html

Guertin, Ghyslaine. 2007. Glenn Gould pluriel. Québec: Momentum.

Hegel, Georg W. F.1995. Cours d'esthétique, trad. J.-P. Lefebvre et V. von Schenck. Paris: Aubier.

Husserl, Edmund. 2001. Phychologie phénoménologique (1925-1928), trad. P. Cabestan, N. Depraz et A. Mazzu, revue par F. Dastur. Paris: Vrin.

—.1972. Recherches logiques, tome 2: Recherches pour la phénoménologie et la théorie de la connaissance (1901), trad. H. Élie, A. L. Kelkel et R. Schérer. Paris: PUF.

Jauss, Hans Robert. 2007. Petite apologie de l'expérience esthétique. Paris: Éditions Allia. 
Katz, Bob. 2002. Mastering Audio; The art and the science. New York: Focal Press.

Lund, Thomas. 2011. Loudness Wars pt. 1, séminaire dans le cadre de Rome Calling, https://www.youtube.com/watch? $=\mathrm{BhA}_{7} \mathrm{Vy}_{3} \mathrm{OPbc}$.

Mantere, Juha Markus. 2006. "The Gould Variations; Technology, philosophy and criticism in Glenn Gould's Thought and Musical Practice». Thèse de doctorat, Université de Tampere.

McClure, John. 1966. "Glenn Gould, The prospects of recording", High Fidelity, vol. 16, no. 4, Avril, https://www.collectionscanada.gc.ca/ glenngould/o28010-4020.01-e.html

Michaud, Alyssa. 2012. "Copies without originals: Manipulation, mediation and mediatization in performance and recording practices», Thèse de doctorat, Université d'Ottawa.

Mill, John Stuart. 2002. Utilitarianism, The Basic Writings of John Stuart Mill. New York: The modern library.

Monsaingeon, Bruno. 1973. "Introduction to The Last Puritan (Le dernier Puritain)". The Glenn Gould Archive (Collections Canada), https://www.collectionscanada.gc.ca/glenngould/o28010-4020.15-e.html.

Moulton, David. 2002. Total Recording, The complete guide to audio production and engineering. États-Unis : KIQ.

Ostwald, Peter. 1997. Glen Gould; The Ecstasy and Tragedy of Genius. New York: W. W. Norton \& Company.

Payzant, Geoffrey. 1978. Glen Gould: Music and Mind. Toronto: Van Nostrand Reinhold.

Platon. Apologie de Socrate. Perseus Digital Library, [17a] à [17c], http://www. perseus.tufts.edu/hopper/text?doc=Perseus\%3Atext\%3A1999.01.0170\%3At ext\%3DApol.

_ 1963. La République, trad. Robert Baccou. Paris: Garnier Frères.

Rojas, Maria Andrea. 2012. "Michel Foucault: la parrêsia, une éthique de la vérité». Thèse de doctorat, Université Paris-Est Créteil.

\section{RÉSUMÉ}

Dans le jargon philosophique habituel, un jugement de type «universel» est un concept toujours valide dans toutes les situations / époques, qui s’applique à tous les individus qu'il a la prétention de catégoriser. Au cours de cet article, nous suggérons que l'ébauche d'une quelconque définition "universelle» de l'art doit se heurter à des problèmes ontologiques (l'essence de l'art) sérieux. Nous proposons qu'il soit plus prudent de s'abstenir de faire de tels jugements en ayant une approche fonctionnaliste pour décrire sans définir le processus créatif ainsi que les œuvres qui y aboutissent. Nous faisons la démonstration de cette approche construite sur un refus conscient de définir l'art, en analysant (avec les catégories des fins et des moyens) le rapport à la technologie du pianiste Glenn Gould. Il s'agit, en quelque sorte, d'un dialogue ouvert avec le lecteur. Finalement, nous aboutissons à la conclusion qu'il peut être pertinent pour le chercheur-créateur (un mélange entre le théoricien et l'artiste qui cherche à valider son art) d'opter pour une esthétique singulière ou d'essayer de constituer une théorie 
universelle de l'art en attaquant le problème ontologique par le biais de la linguistique (une suggestion, sans garanties). En dehors de notre approche fonctionnaliste, qui par rigueur intellectuelle peut tendre vers un relativisme insatisfaisant, il s'agit des seuls choix prometteurs qui ne se limitent pas aux analyses historiques ou aux analyses d'œuvres individuelles (le paradigme actuel de l'enseignement théorique universitaire en musique) auxquels nous avons pensé.

\begin{abstract}
In the usual philosophical jargon, a judgment qualified as "universal" is a concept valid at all times and in all contexts or periods, that apply to all individuals it intends to classify. In this article, I argue that any tentative "universal" definition of art is fraught with serious ontological problems (regarding its essence). I propose therefore that it would be more prudent to refrain from making such attempts, and to adopt instead a functional approach in describing, without defining, the creative processes, as well as the resulting musical works. I demonstrate this approach, which is built on a deliberate refusal to define art, by engaging in an open dialogue with the reader, analyzing (in terms of means and ends) how the pianist Glenn Gould related to technology. I conclude that it could be fruitful for the creator-searcher (the artist exploring the craft through theory) to choose a specific esthetics or to develop a universal theory of art through addressing the ontological problems with the help of linguistics (a suggestion with no guarantees). Indeed, apart from the functionalist approach, which may become unsatisfactory relativism, due to intellectual rigour, these avenues are the only promising ones that are not limited to historical or musical analyses of individual works (analyses that are the basis of the theoretical musical training in our universities).
\end{abstract}

\title{
BIOGRAPHIE
}

En 2011, après avoir terminé un baccalauréat en philosophie à l'Université Laval (2011), Dragos Chiriac entame des études universitaires en réalisation audionumérique. Après un certificat (2011) et une maîtrise sur mesure en réalisation audionumérique (2013), Dragos poursuit ses études au doctorat en musicologie, volet recherche-création sous la direction de Sophie Stévance et de Serge Lacasse : ses spécialités sont la compression (réduction de marge dynamique), la sonie et le matriçage. En plus de développer une méthode consistant à distribuer l'étape de la réduction de la gamme dynamique (normalement effectuée au matriçage) sur l'ensemble des étapes de la réalisation phonographique, Dragos compose et réalise la musique de plusieurs groupes comme Men I Trust, Ghostly Kisses et Geoffroy Sauvé (voir www.dragos.ca pour la liste complète). 